\title{
The Dirac Operator with Highly Singular Potentials
}

\author{
D. B. Pearson
}

Department of Applied Mathematics, University of Hull, Hull HU6 7RX, England

\begin{abstract}
Time-dependent scattering theory for a Dirac particle with highly singular potential is developed. Criteria for asymptotic completeness of wave operators are obtained, and an example is given of a potential which violates asymptotic completeness and the unitarity of the scattering operator. (Completeness breaks down for a regular sequence of values of the coupling constant.)
\end{abstract}

\section{Introduction}

This paper sets out to develop the scattering theory of a relativistic Dirac particle with potentials which may be highly singular. (For related work and references on the Dirac operator see $[1-4]$.) The aim is to bring aspects of the theory relating to asymptotic completeness of wave operators and spectral properties of the Hamiltonian into line with results for the corresponding non-relativistic Schrödinger problem, and in particular with the results of [9-11]. (The existence of wave operators for highly singular potentials of short range is a consequence of the argument of Kupsch and Sandhas [5], cf. [1] for the necessary estimates.)

Section 2 deals briefly with the general theory of scattering by a potential which is singular on some closed, bounded set $\Sigma$ of measure zero. As in the Schrödinger case, the Hilbert space decomposes into two orthogonal subspaces, consisting respectively of states in which, for large times, the particle with probability 1 approaches $\Sigma$, its kinetic energy becoming unbounded, and states in which the particle escapes to infinity. Apart from technical details (for example only a local condition need be imposed on the potential away from its singularities) this treatment follows closely that of [10]. For short range potentials, (strong) asymptotic completeness corresponds to the absence of states of the first kind.

We consider that the setting up of criteria for completeness logically precedes further consideration of the more unusual situation where completeness is violated. Section 3 proves completeness for a wide range of spherically symmetric short range potentials, singular or non-singular, and establishes for these potentials the absence of singular continuous spectrum. The main results, in Theorem 3, apply in 
particular to $V(r)=g / r^{K}$ near $r=0$, where $g$ and $K$ are arbitrary. This work parallels that of [9] in the Schrödinger case, though apart from their common feature of depending on estimates near the origin of solutions of the time independent eigenvalue equation the two methods are different.

It would also be possible to deal with non-singular short range potentials which are not necessarily spherically symmetric (see [2]). For example, the results of Nenciu [7] allow one to conclude the completeness of wave operators for potentials satisfying $|V(\boldsymbol{r})| \leqq \mu / r(\mu<1)$ near $r=0$, and have been extended by the same author to include potentials dominated by a multicentre Coulomb potential ([8]). But it seems that such results will not in general be possible for highly singular potentials, the likelihood being that for cases in which the deficiency indices are infinite completeness will depend on which extension of the Hamiltonian is taken.

Section 4 provides an example of a short range spherical potential for which completeness breaks down, due to the presence of states in which the particle is asymptotically absorbed into the origin. In contrast to [11], an explicit formula for $V(r)$ is obtained, and a spectral analysis carried out for all values of the coupling constant. The situation differes from the non-relativistic case in the following respects :

i) We can have $V(r) \geqq 0$ for all $r$ (which would imply completeness in the nonrelativistic case).

ii) Breakdown of completeness occurs for discrete values of the coupling constant, which recur at regular intervals.

iii) $V(r)$ is "less singular" than in the non-relativistic case, and may be found such that

$\int_{0}^{1} r^{\varepsilon} V(r) d r<\infty \quad$ and $\quad V(r)<\frac{\text { const }}{r^{2+\varepsilon}}, \quad \varepsilon>0$.

A reasonable conjecture would appear to be that, for arbitrary singular potentials, completeness holds for "almost all" values of the coupling constant.

\section{Scattering with Absorption at Local Singularities}

Let $H_{0}$ denote the free Dirac Hamiltonian, the unique self-adjoint extension, in $\left[L_{2}\left(\mathbb{R}^{3}\right)\right]^{4}$, of the differential operator $\boldsymbol{\alpha} \cdot \boldsymbol{p}+m \beta$. We assume that the potential $V_{i j}(\boldsymbol{r})$ is $L_{3}^{(\text {loc })}$ in the complement of some closed, bounded set $\Sigma$ of measure zero.

Let $\hat{H}$ denote the differential operator $\boldsymbol{\alpha} \cdot \boldsymbol{p}+m \beta+V$, defined on $C_{0}^{\infty}\left(\mathbb{R}^{3} \backslash \Sigma\right)$, the $C^{\infty}$ functions with compact support contained in the complement of $\Sigma$; and let $H$ denote a self-adjoint extension, in $\left(L_{2}\left(\mathbb{R}^{3}\right)\right]^{4}$, of $\hat{H}$. We first note a number of results which we shall need in this section.

i) $D(H) \subseteq D^{(\text {loc })}\left(H_{0}\right) \cap D^{(\text {loc })}(V)$.

$\left(\right.$ Here, $D^{(\text {loc) }}(T) \equiv\left\{F ; F \in\left[L_{2}\left(\mathbb{R}^{3}\right)\right]^{4}\right.$ and $\left.\left.\varrho F \in D(T) \forall \varrho \in C_{0}^{\infty}\left(\mathbb{R}^{3} \backslash \Sigma\right).\right\}\right)$

Proof. Essentially contained in [3]; cf. [10], Section II.

ii) $\varrho \in C_{0}^{\infty}\left(\mathbb{R}^{3} \backslash \Sigma\right) \Rightarrow \varrho(H+i)^{-2}$ is Hilbert-Schmidt.

Proof. Certainly $\varrho\left(H_{0}+i\right)^{-2}$ is Hilbert-Schmidt. Applying the commutation relation $\left[\varrho,\left(H_{0}+i\right)^{-1}\right]=\left(H_{0}+i\right)^{-1} \varrho^{\prime}\left(H_{0}+i\right)^{-1}$ where $\varrho^{\prime}=-i \alpha_{k} \frac{\partial \varrho}{\partial x_{k}}$, we see that 
$\left(H_{0}+i\right)^{-1} \varrho\left(H_{0}+i\right)^{-1}$ is Hilbert-Schmidt. Now choose $\varrho_{1} \in C_{0}^{\infty}\left(\mathbb{R}^{3} \backslash \Sigma\right)$, real, with $\varrho_{1}(\boldsymbol{r}) \equiv 1$ on the support of $\varrho$.

Then (i) $\Rightarrow\left(H_{0} \pm i\right) \varrho_{1}(H \pm i)^{-1}$ is bounded, by the closed graph theorem.

Hence $(H+i)^{-1} \varrho(H+i)^{-1}=\left[\left(H_{0}-i\right) \varrho_{1}(H-i)^{-1}\right]^{*}\left(H_{0}+i\right)^{-1} \varrho\left(H_{0}+i\right)^{-1}\left[\left(H_{0}\right.\right.$ $\left.+i) \varrho_{1}(H+i)^{-1}\right]$, and is Hilbert-Schmidt. Similarly, $(H+i)^{-1} \varrho^{\prime}(H+i)^{-1}$ is HilbertSchmidt. The result now follows on writing $\varrho(H+i)^{-2}=\left[\varrho,(H+i)^{-1}\right](H+i)^{-1}$ $+(H+i)^{-1} \varrho(H+i)^{-1}$ and using the commutation relation

$\left[\varrho,(H+i)^{-1}\right]=(H+i)^{-1} \varrho^{\prime}(H+i)^{-1}$.

(iii) $\varrho \in C_{0}^{\infty}\left(\mathbb{R}^{3} \backslash \Sigma\right) \Rightarrow \varrho(H+i)^{-4}$ is of trace class.

Proof. With $\varrho_{1}$ as above,

$$
\begin{aligned}
& (H+i)^{-1} \varrho(H+i)^{-3}=(H+i)^{-1} \varrho \varrho_{1}(H+i)^{-1}(H+i)^{-2} \\
& \quad=(H+i)^{-1} \varrho\left\{(H+i)^{-1} \varrho_{1}+(H+i)^{-1} \varrho_{1}^{\prime}(H+i)^{-1}\right\}(H+i)^{-2}
\end{aligned}
$$

and is of trace class. Similarly, $(H+i)^{-1} \varrho^{\prime}(H+i)^{-3}$ is of trace class. The result now follows on writing

$$
\varrho(H+i)^{-4}=\left((H+i)^{-1} \varrho+\left[\varrho,(H+i)^{-1}\right]\right)(H+i)^{-3} .
$$

(iv) $\varrho \in C_{0}^{\infty}\left(\mathbb{R}^{3} \backslash \Sigma\right) \Rightarrow \varrho E_{|H|<c}$ is of trace class.

Proof. The result follows directly from (iii), since $(H+i)^{4} E_{|H|<c}$ is bounded.

We now define subspaces $M_{\Sigma}^{ \pm}, M_{\infty}^{ \pm}, N_{\infty}^{ \pm}, N_{f}^{ \pm}$of $M_{\text {a.c. }}(H)$ as follows, where $\Sigma \leqq\{\boldsymbol{r} ;|\boldsymbol{r}| \leqq R\}, g \in M_{\text {a.c. }}(H)$,

$g \in M_{\Sigma}^{ \pm}$iff $\quad \underset{t \rightarrow \pm \infty}{s-\lim _{t \rightarrow \infty}} E_{|\boldsymbol{r}|>a} e^{-i H t} g=0, \forall a>R$,

$g \in M_{\infty}^{ \pm} \quad$ iff $\quad \underset{t \rightarrow \pm \infty}{s-\lim _{t \rightarrow \infty}} E_{|\boldsymbol{r}|<a} e^{-i H t} g=0, \forall a>R$,

$g \in N_{\infty}^{ \pm} \quad$ iff $\quad \lim _{t \rightarrow \pm \infty} E_{\left|H_{0}\right|<b} e^{-i H t} g=0, \forall b>0$,

$g \in N_{f}^{+}$(respectively $N_{f}^{-}$) iff, given any $\varepsilon>0$,

$\exists \beta, T>0$ such that $\left\|E_{\left|H_{0}\right|>b} e^{-i H t} g\right\|<\varepsilon$,

$\forall b>\beta, t>T$ (respectively $t<-T$ ).

These subspaces are independent of the value of $R$.

Theorem 1. (a) $M_{\Sigma}^{+} \perp M_{\infty}^{+} ; M_{\Sigma}^{-} \perp M_{\infty}^{-}$.

(b) $M_{\Sigma}^{ \pm}=N_{\infty}^{ \pm} ; M_{\infty}^{ \pm}=N_{f}^{ \pm}$.

(c) Denoting by $P_{\Sigma}^{ \pm}$and $P_{\infty}^{ \pm}$the orthogonal projections onto $M_{\Sigma}^{ \pm}$and $M_{\infty}^{ \pm}$ respectively, we have

$$
\begin{aligned}
& P_{\Sigma}^{ \pm}=s-\lim _{t \rightarrow \pm \infty} e^{i H t} E_{|\boldsymbol{r}|<a} e^{-i H t} P_{\text {a.c. }}(H), \\
& P_{\infty}^{ \pm}=s-\lim _{t \rightarrow \pm \infty} e^{i H t} E_{|\boldsymbol{r}|>a} e^{-i H t} P_{\text {a.c. }}(H),
\end{aligned}
$$

where $a>R$ and $P_{\text {a.c. }}(H)$ is the projection onto $M_{\text {a.c. }}(H)$.

(d) $M_{\text {a.c. }}(H)=M_{\Sigma}^{ \pm} \oplus M_{\infty}^{ \pm}$and the subspaces reduce $H$. 
Proof. See [10], Theorem 2. The proof of (1) and (2) depends on the existence of s-lim $e^{i H t} \varrho e^{-i H t} E_{|H|<c} P_{\text {a.c. }}(H)$, where now $\varrho(\boldsymbol{r}) \equiv 1$ for $|\boldsymbol{r}|$ sufficiently large and supp $\cdot \varrho \subset \mathbb{R}^{3} \backslash \Sigma$. This result follows from a trace theorem $([12])$, since (iv) above $\Rightarrow \varrho^{\prime} E_{|H|<c}$ is of trace class. The remainder of the proof follows [10] precisely.

We also have

Theorem 2. Suppose that, for $|\boldsymbol{r}|>a>R$ and for some $\varepsilon>0$,

$$
|V(\boldsymbol{r})| \leqq \text { const }|\boldsymbol{r}|^{-(2+\varepsilon)} \text {. }
$$

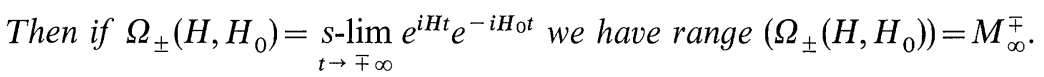

Proof. See [10], Theorem 3. With $\varrho$ as in the proof of Theorem 1, we have to prove the existence of

$\underset{t \rightarrow \pm \infty}{s-\lim } e^{i \tilde{H} t} \varrho e^{-i H t} E_{|H|<c} P_{\text {a.c. }}(H)$, where $\tilde{H}$ is a self-adjoint extension corresponding to a potential

$$
\begin{aligned}
\tilde{V}(\boldsymbol{r}) & =V(\boldsymbol{r}), \quad|\boldsymbol{r}|>a \\
& =0, \quad \text { otherwise. }
\end{aligned}
$$

(Note that $\varrho(V-\tilde{V})$ vanishes for suitable $\varrho$. .)

The asymptotic completeness of $\Omega_{ \pm}\left(\tilde{H}, H_{0}\right)$ follows from [2].

Corollary. If $V(\boldsymbol{r})=V(|\boldsymbol{r}|) \delta_{i j}$ is spherically symmetric, and the self-adjoint extension $H$ is taken to commute with total angular momentum, $|V(r)| \leqq$ const $\cdot r^{-(1+\varepsilon)}$ is sufficient for the conclusion of Theorem 2.

Remark. Theorem 2 shows that (strong) asymptotic completeness is equivalent to $M_{\Sigma}^{ \pm}=\{0\}$. This forms the basis for the proofs of completeness for singular potentials given in the following section.

\section{Criteria for Asymptotic Completeness, and Spectral Properties}

A state having quantum numbers $J, M$ of total angular momentum, and parity $(-1)^{J+(1 / 2) \omega}(\omega= \pm 1)$ may be represented (with a suitable choice of the matrix $\beta$ ) by the 4-spinor

$$
r^{-1}\left(\frac{F(r) Y_{l J}^{M}}{i G(r) Y_{l^{\prime} J}^{M}}\right), \quad \text { where } \quad l=J+\frac{1}{2} \omega, l^{\prime}=J-\frac{1}{2} \omega,
$$

and $Y_{l J}^{M}, Y_{l^{\prime} J}^{M}$ are appropriate functions of polar angles and of $z$-component of spin, combining a spherical harmonic of order $L$ with spin angular momentum $\frac{1}{2} \hbar$ (see e.g. [6], Chapter XX).

If the potential $V(r)$ [i.e. $V_{i j}(\boldsymbol{r})=V(|\boldsymbol{r}|) \delta_{i j}$ ] is spherically symmetric, we may consider self-adjoint extensions of $\boldsymbol{\alpha} \cdot \boldsymbol{p}+m \beta+V(r)$ which leave each $J, M, \omega$ subspace invariant. In each subspace the Hamiltonian will then be some self-adjoint 
extension, acting now in $\left[L_{2}(0, \infty)\right]^{2}$, of the differential operator $\tau$ defined on $C^{\infty}$ functions of compact support in $(0, \infty)$, where

$$
\tau\left(\begin{array}{l}
F(r) \\
G(r)
\end{array}\right)=\left(\begin{array}{c}
-\frac{d G}{d r}+\omega \frac{\left(J+\frac{1}{2}\right)}{r} G+(V(r)+m) F \\
\frac{d F}{d r}+\omega \frac{\left(J+\frac{1}{2}\right)}{r} F+(V(r)-m) G
\end{array}\right) .
$$

To investigate spectral properties and the related question of asymptotic completeness, we first consider self-adjoint extensions $H_{a}$, acting in $L_{2}(0, a)$ of $\tau$ defined on $C^{\infty}$ functions of compact support in $(0, a)$. In constructing the corresponding resolvent $\left(H_{a}-\lambda\right)^{-1}$, we shall estimate near $r=0$ solutions $\left(\begin{array}{l}\phi \\ \psi\end{array}\right)$ of the equations

$$
\tau\left(\begin{array}{l}
\phi \\
\psi
\end{array}\right)=\lambda\left(\begin{array}{l}
\phi \\
\psi
\end{array}\right), \quad(\lambda \text { real })
$$

If solutions $\left(\begin{array}{l}\phi_{1} \\ \psi_{1}\end{array}\right),\left(\begin{array}{l}\phi_{2} \\ \psi_{2}\end{array}\right)$ may be found with

$$
\phi_{1} \psi_{2}-\phi_{2} \psi_{1}=1
$$

and

$$
\left(\begin{array}{l}
\phi_{1} \\
\psi_{1}
\end{array}\right) \in\left[L_{2}(0, a)\right]^{2},
$$

we may formally define a resolvent by

$$
\left(H_{a}-\lambda\right)^{-1}\left(\begin{array}{l}
F \\
G
\end{array}\right)=\left(\begin{array}{l}
u \\
v
\end{array}\right)
$$

with

$$
\left.\begin{array}{rl}
u(r)= & -\phi_{1}(r) \int_{r}^{a}\left(\phi_{2}(t) F(t)+\psi_{2}(t) G(t)\right) d t \\
& -\phi_{2}(r) \int_{0}^{r}\left(\phi_{1}(t) F(t)+\psi_{1}(t) G(t)\right) d t \\
v(r)= & -\psi_{1}(r) \int_{r}^{a}\left(\phi_{2}(t) F(t)+\psi_{2}(t) G(t)\right) d t \\
& -\psi_{2}(r) \int_{0}^{r}\left(\phi_{1}(t) F(t)+\psi_{1}(t) G(t)\right) d t
\end{array}\right\}
$$

This corresponds to the boundary condition $\psi_{2}(a) u(a)-\phi_{2}(a) v(a)=0$, at $\quad r=a$.

In (7), $\left(\begin{array}{l}u \\ v\end{array}\right)$ may be verified to be a solution of $(\tau-\lambda)\left(\begin{array}{l}u \\ v\end{array}\right)=\left(\begin{array}{l}F \\ G\end{array}\right)$. Moreover, if $\left(\begin{array}{l}u \\ v\end{array}\right) \in C_{0}^{\infty}((0, a))$ and $\left(\begin{array}{l}F \\ G\end{array}\right)=(\tau-\lambda)\left(\begin{array}{l}u \\ v\end{array}\right)$ then $(7)$ is satisfied. 
If on the other hand no solution to (4) exists in $\left[L_{2}(0, a)\right]^{2}$, apart from the trivial solution, then $\lambda$ lies in the essential spectrum of $H_{a}$, and the resolvent is unbounded. A potential for which this happens is given in Section 4. We shall obtain conditions on $V(r)$ which will guarantee that the mapping defined by (7) is Hilbert-Schmidt, in which case the resolvent of $H_{a}$ is compact.

Given a non-trivial solution $\left(\begin{array}{l}\phi \\ \psi\end{array}\right)$ of (4), we define $\theta(r)$, up to a multiple of $2 \pi$, by

$$
\left.\begin{array}{l}
\phi(r)=R(r) \cos \theta(r) \\
\psi(r)=R(r) \sin \theta(r)
\end{array}\right\}
$$

More exactly, $\theta(r)$ is defined to satisfy

$$
\frac{d \theta}{d r}=\frac{\phi \frac{d \psi}{d r}-\psi \frac{d \phi}{d r}}{\left(\phi^{2}+\psi^{2}\right)} .
$$

Equation (8) defines the Prüfer transformation for the Dirac equation.

From (3), (4) we have

$$
\phi \frac{d \psi}{d r}-\psi \frac{d \phi}{d r}=\frac{2 \omega(J+1 / 2)}{r} \phi \psi-(\lambda-m-V) \phi^{2}-(\lambda+m-V) \psi^{2},
$$

which on substituting (8) on the r.h.s. becomes

$$
\frac{d \theta}{d r}=\frac{\omega(J+1 / 2)}{r} \sin 2 \theta+m \cos 2 \theta-(\lambda-V)
$$

We also have

$$
\frac{d}{d r} R^{2}=2 \phi \frac{d \phi}{d r}+2 \psi \frac{d \psi}{d r}=\frac{2 \omega(J+1 / 2)}{r}\left(\psi^{2}+\phi^{2}\right)+4 m \phi \psi,
$$

which becomes

$$
\frac{d}{d r}(\log R)=\frac{-\omega(J+1 / 2)}{r} \cos 2 \theta+m \sin 2 \theta .
$$

Equations (9) and (10) may be used to estimate $R$ and $\theta$ near $r=0$. From (10) we have

$$
|\log R|<\mathrm{const}+|\omega(J+1 / 2) \log r|,
$$

so that

$$
R=0\left(r^{-(J+1 / 2)}\right) \quad(r \rightarrow 0)
$$

independently of the potential.

We can obtain more precise estimates in two cases, which include examples both of singular and non-singular potentials.

Case I. $|r V(r)|<g \quad 0<g<J+1 / 2$.

First choose $g_{1}$ with $g<g_{1}<J+1 / 2$. 
Then from (9) we see that, for $r$ sufficiently small,

$$
\frac{d \theta}{d(-r)}<0 \quad \text { whenever } \quad \omega \sin 2 \theta \geqq g_{1}(J+1 / 2)^{-1},
$$

whereas

$$
\frac{d \theta}{d(-r)}>0 \quad \text { whenever } \quad \omega \sin 2 \theta \leqq-g_{1}(J+1 / 2)^{-1} .
$$

This enables us to deduce that, for $r$ sufficiently small,

$$
\begin{aligned}
& \sin ^{2} 2 \theta<g_{1}^{2}(J+1 / 2)^{-2}, \quad \text { so that either } \\
& \omega \cos 2 \theta>\left(1-g_{1}^{2}(J+1 / 2)^{-2}\right)^{1 / 2}
\end{aligned}
$$

or

$$
\omega \cos 2 \theta<-\left(1-g_{1}^{2}(J+1 / 2)^{-2}\right)^{1 / 2},
$$

either the first inequality always holding, for small $r$, or the second.

Suppose first that (12) holds. Then on integrating (10) we find $R>$ const $r^{-\beta_{1}}$ as $r \rightarrow 0$, where $\beta_{1}=\left((J+1 / 2)^{2}-g_{1}^{2}\right)^{1 / 2}$. Take first the case $\omega=+1$. Then (12) $\Rightarrow|\cos \theta|$ $>$ const $>0$, so that, using (8), we can take $a$ sufficiently small that

$$
|\phi(r)|>\text { const } \cdot r^{-\beta_{1}} \quad(0<r<a)
$$

Let $\left(\begin{array}{l}\phi^{\prime} \\ \psi^{\prime}\end{array}\right)$ be a second solution of (4), satisfying $\phi \psi^{\prime}-\psi \phi^{\prime}=1$.

We have

$$
\frac{d \phi^{\prime}}{d r}=-\omega \frac{(J+1 / 2)}{r} \phi^{\prime}+\frac{(\lambda+m-V)\left(1+\psi \phi^{\prime}\right)}{\phi},
$$

and substituting from (4) for $\frac{(\lambda+m-V)}{\phi} \psi$ gives $\frac{d \phi^{\prime}}{d r}=\frac{(\lambda+m-V)}{\phi}+\frac{\phi^{\prime}}{\phi} \frac{d \phi}{d r}$, so that

$$
\phi^{\prime}(r)=\phi(r)\left(\text { const }+\int_{0}^{r} \frac{(\lambda+m-V(t))}{\phi^{2}(t)} d t\right)
$$

Hence, corresponding to every solution $\left(\begin{array}{l}\phi \\ \psi\end{array}\right)$ of (4) satisfying (12) we can find a solution $\left(\begin{array}{l}\tilde{\phi} \\ \tilde{\psi}\end{array}\right)$ of (4), such that $\tilde{\phi}(r)=\phi(r) \int_{0}^{r} \frac{(\lambda+m-V(t))}{\phi^{2}(t)} d t$.

For this solution, (13) gives $\tilde{\phi}=0\left(r^{2 \beta_{1}} \phi\right)$ as $r \rightarrow 0$. If $\tilde{\phi}$ satisfies (12) we can construct a further solution which is $0\left(r^{4 \beta_{1}} \phi\right)$ as $r \rightarrow 0$, and ultimately [because of (11)] we must, continuing in this way arrive at a solution $\left(\begin{array}{l}\phi_{1} \\ \psi_{1}\end{array}\right)$ for which (12) and (13) are not satisfied. $\left(\begin{array}{l}\phi_{1} \\ \psi_{1}\end{array}\right)$ must then satisfy $\left(12^{\prime}\right)$. Using (10), we then have

$$
\begin{aligned}
& R=0\left(r^{\beta_{1}}\right) \quad \text { as } \quad r \rightarrow 0, \quad \text { so that } \\
& \phi_{1}(r)=0\left(r^{\beta_{1}}\right) \quad \text { and } \quad \psi_{1}(r)=0\left(r^{\beta_{1}}\right) .
\end{aligned}
$$


From (11) we also have $\phi_{2}(r)=0\left(r^{-(J+1 / 2)}\right)$ and $\psi_{2}(r)=0\left(r^{-(J+1 / 2)}\right)$ for any solution $\left(\begin{array}{l}\phi_{2} \\ \psi_{2}\end{array}\right)$, and the operator defined by (7) is Hilbert-Schmidt provided $g^{2}<2 J$. The case $\omega=-1$ is similar, with $\phi$ replaced by $\psi$ in (13).

In the case $V(r)=g / r,|g|<(J+1 / 2)$, eliminating $\psi(r)$ from (4) and making the change of variables $z=\int(\lambda+m-V) d r$ we find solutions $\phi_{1}(r)=0\left(r^{\beta}\right), \psi_{1}=0\left(r^{\beta}\right)$ and $\phi_{2}(r)=0\left(r^{-\beta}\right), \psi_{2}(r)=0\left(r^{-\beta}\right)$, with $\beta=\left((J+1 / 2)^{2}-g^{2}\right)^{1 / 2}$. For $|g|=(J+1 / 2),\left(\begin{array}{l}\phi_{2} \\ \psi_{2}\end{array}\right)$ has a logarithmic singularity. In both cases the operator defined by (7) is HilbertSchmidt.

Case II. $|r V(r)|>g, g>(J+1 / 2)$, and $(r V(r))^{-1}$ of bounded variation. In (9), let us write

$$
\begin{aligned}
\Delta(r) & =\sin 2 \theta+\frac{\omega r}{(J+1 / 2)}[m \cos 2 \theta-(\lambda-V)] \\
& =Q(r)+Z(r),
\end{aligned}
$$

where

$$
Q(r)=\sin 2 \theta+\frac{m \omega r \cos 2 \theta}{(J+1 / 2)}
$$

and

$$
Z(r)=-\frac{\omega r(\lambda-V)}{(J+1 / 2)}
$$

so that (9) becomes

$$
\frac{d \theta}{d r}=\frac{\omega(J+1 / 2)}{r} \Delta(r)
$$

From (10) we have

$$
[\log R]_{r}^{a}=-\omega(J+1 / 2) \int_{r}^{a} \frac{\cos 2 \theta}{r} d r+0(1),
$$

and since $\frac{d \theta}{d r} \neq 0$ we may make a change of variable to obtain

$$
[\log R]_{r}^{a}=-\int_{\theta(r)}^{\theta(a)} \frac{\cos 2 \theta}{\Delta} d \theta+0(1) .
$$

In (17) we substitute

$$
\frac{1}{\Delta}=\frac{1}{Z+Q}=\frac{1}{Z}-\frac{Q}{Z^{2}}+\ldots+\frac{(-1)^{n} Q^{n}}{Z^{n+1}}+\frac{(-1)^{n+1} Q^{n+1}}{Z^{n+1} \Delta}
$$

and estimate each of the resulting terms.

For example, $\int \frac{\cos 2 \theta}{Z} d \theta=\left[\frac{\sin 2 \theta}{2 Z}\right]-\int \sin 2 \theta d\left(\frac{1}{2 Z}\right)=0(1)$ since $|Z|>1$ and $Z^{-1}$ is of bounded variation.

Moreover, $Q^{m} \cos 2 \theta$ is a linear combination of terms having the form $r^{k}(\cos 2 \theta)^{k+1}(\sin 2 \theta)^{m-k}$ with $k=0,1,2, \ldots, m$. 
For $k \geqq 1$, we have

$\left|\int_{\theta(r)}^{\theta(a)} \frac{r^{k}(\cos 2 \theta)^{k+1}(\sin 2 \theta)^{m-k}}{Z^{m+1}} d \theta\right|$

$\leqq$ const $\int_{r}^{a} \frac{r^{k-1}}{Z^{m+1}} \Delta d r=0(1), \quad$ since $\Delta / Z^{m+1}$

is bounded.

For $k=0$ we have $\int_{0}^{\pi}(\cos 2 \theta)(\sin 2 \theta)^{m} d \theta=0$, so that we can write $(\cos 2 \theta)(\sin 2 \theta)^{m}$ $=\frac{d I}{d \theta}$ where $I$ is bounded, giving

$$
\int \frac{1}{Z^{m+1}} \frac{d I}{d \theta} d \theta=\left[I / Z^{m+1}\right]-\int I d\left(\frac{1}{Z^{m+1}}\right)=0(1)
$$

since $1 / Z^{m+1}$ is of bounded variation.

We have, finally, from (17),

$$
\begin{aligned}
{[\log R]_{r}^{a} } & =(-1)^{n} \int_{\theta(r)}^{\theta(a)} \frac{Q^{n+1}(\cos 2 \theta) d \theta}{Z^{n+1} \Delta}+0(1) \\
& =(-1)^{n} \omega(J+1 / 2) \int_{r}^{a} \frac{Q^{n+1} \cos 2 \theta}{r Z^{n+1}} d r+0(1) .
\end{aligned}
$$

Since $|Q / Z|<$ const $<1$ (for $r$ small), we can take $n$ sufficiently large that $[\log R]_{r}^{a}=0(\varepsilon \log r), \quad$ or $R=0\left(r^{-\varepsilon}\right), \quad r \rightarrow 0, \quad \varepsilon$ arbitrarily small.

In this case we have, for all solutions $\left(\begin{array}{l}\phi \\ \psi\end{array}\right)$ of $(4), \phi=0\left(r^{-\varepsilon}\right), \psi=0\left(r^{-\varepsilon}\right)$, and the operator defined by (7) is Hilbert-Schmidt.

Remark 1. With further conditions on $V(r)$, one may obtain $\phi(r)=(a+0(1))$ $\cdot \cos \int_{r}^{a} V(t) d t+(b+0(1)) \sin \int_{r}^{a} V(t) d t$, which applies in particular to $V(r)=\frac{g}{r^{n}}, n>1$. A similar estimate holds for $\psi(r)$.

Remark 2. A perturbation $W(r)$ may be added to $V(r)$, in which case a solution $\left(\begin{array}{l}\Phi \\ \Psi\end{array}\right)$ of (4) with the perturbed potential is given in terms of the original solutions with potential $V(r)$ by the integral equations

$$
\begin{aligned}
\Phi(r)= & \phi_{1}(r)+\phi_{1}(r) \int_{r}^{a}\left(\phi_{2}(t) W(t) \Phi_{1}(t)+\psi_{2}(t) W(t) \Psi_{1}(t)\right) d t \\
& +\phi_{2}(r) \int_{0}^{r}\left(\phi_{1}(t) W(t) \Phi_{1}(t)+\psi_{1}(t) W(t) \Psi_{1}(t)\right) d t \\
\Psi(r)= & \psi_{1}(r)+\psi_{1}(r) \int_{r}^{a}\left(\phi_{2}(t) W(t) \Phi_{1}(t)+\psi_{2}(t) W(t) \Psi_{1}(t)\right) d t \\
& +\psi_{2}(r) \int_{0}^{r}\left(\phi_{1}(t) W(t) \Phi_{1}(t)+\psi_{1}(t) W(t) \Psi_{1}(t)\right) d t .
\end{aligned}
$$


For a suitable class of perturbations these equations may be iterated to derive estimates for $\left(\begin{array}{l}\Phi \\ \Psi\end{array}\right)$ similar to those for $\left(\begin{array}{l}\phi_{1} \\ \psi_{1}\end{array}\right)$. Extending in this way the class of potentials with which we can deal, we state the following:

Theorem 3. Let $V(r)$ satisfy either (i) $V(r)=\frac{g}{r}+W(r)$, where $0 \leqq g \leqq(J+1 / 2)$ and $\int_{0}^{a}|W(r)| d r<\infty\left(\int_{0}^{a}|W(r) \log r| d r<\infty \quad\right.$ for $\left.\quad|g|=(J+1 / 2)\right)$

or (ii) $V(r)=V_{0}(r)+W(r)$, where $\left|r V_{0}(r)\right|<g<\sqrt{2 J}$. and

$$
\int_{0}^{a} r^{\sqrt{\left((J+1 / 2)^{2}-g^{2}\right)}-(J+1 / 2)}|W(r)| d r<\infty
$$

or (iii) $V(r)=V_{0}(r)+W(r)$, where $\left|r V_{0}(r)\right|>g>(J+1 / 2),\left(r V_{0}(r)\right)^{-1}$ has bounded variation, and

$$
\int_{0}^{a} r^{-\varepsilon}|W(r)| d r<\infty \quad \text { for some } \varepsilon>0
$$

Then solutions of (4) exist, where $\tau$ is defined by (3), such that (6) and (7) define the resolvent of a self-adjoint extension $H_{a}$ of $\tau$, acting in $L_{2}(0, a)$. This resolvent is HilbertSchmidt and the spectrum of $H_{a}$ is purely discrete. If in addion $V(r)$ is defined for $0<r$ $<\infty$ and satisfies $\int_{c}^{\infty} r^{\varepsilon}|V(r)| d r<\infty$ for all $c>0$ and some $\varepsilon>0$, the wave operators $\Omega_{ \pm}\left(H, H_{0}\right)$ corresponding to every self adjoint extension $H$ of $\tau$ acting in $L_{2}(0, \infty)$, where $\mathrm{H}_{0}$ is the Free Dirac Hamiltonian, are asymptotically complete.

Proof. It is straightforward to verify that $\left(H_{a}-\lambda\right)^{-1}$ is self-adjoint and that the inverse of $\left(H_{a}-\lambda\right)^{-1}$ is a self-adjoint extension of $\tau-\lambda$ acting in $L_{2}(0, a)$. The Hilbert-Schmidt property and discrete spectrum follow from previous arguments.

Now if $\varrho$ is $C^{\infty}$ such that $\varrho(r) \equiv 1$ near $r=0$ and $\varrho(r)=0$ for $r>(1 / 2) a$, we have

$$
f \in D(H) \Rightarrow \varrho f \in D\left(H_{a}\right) \quad \text { [cf. (i) of Section 2]. }
$$

Hence $\left(H_{a}-\lambda\right) \varrho(H-i)^{-1}$ is bounded (by the closed graph theorem) and the compactness of $\left(H_{a}-\lambda\right)^{-1}$ implies that $\varrho(H-i)^{-1}$ is compact. If $g \in M_{\text {a.c. }}(H)$ we then have $s$-lim $\varrho e^{-i H t}(H-i)^{-1} g=0$, and since the domain of $H$ is dense it follows that $M_{ \pm}^{\Sigma}(H)$ is just the zero element, which suffices, by the results of Section (2), to prove asymptotic completeness.

Remark. The above theorem applies in particular to $V(r)=g / r^{K}$ near $r=0$, where $g$ and $K$ are arbitrary. Essential self-adjointness holds only with $K<1$ or with $K=1$, $|g| \leqq \sqrt{3 / 2}($ see $[4,8])$.

\section{A Potential Violating Asymptotic Completeness}

We have seen that the "normal" situation for self-adjoint extensions in $\left[L_{2}(0, a)\right]^{2}$ of the differential operator $\tau$ defined by (3) is that the spectrum is purely discrete. We now obtain a potential which gives rise to absolutely continuous spectrum. 
The potential $V(r)$ will be constructed from a series of $\delta$-type singularities. We first consider the behaviour near $r=r_{0}$ of the solution of (4) for a potential which "approximates" $g \delta\left(r-r_{0}\right)$.

Let $V(r)=0,\left|r-r_{0}\right|>\varepsilon(\varepsilon<a<1)$ and suppose

$$
\int_{r_{0}-\varepsilon}^{r_{0}+\varepsilon} V(r) d r=g \text {. }
$$

Integrating (9) we find, as $\varepsilon \rightarrow 0$,

$$
\theta\left(r_{0}+\varepsilon\right)-\theta\left(r_{0}-\varepsilon\right)=g+0\left(\frac{\varepsilon}{r_{0}}\right)
$$

Now fixing the value of $\theta\left(r_{0}+\varepsilon\right), \theta$ is also a differentiable function of $\lambda$, satisfying the integral equation

$$
\begin{aligned}
& \frac{d \theta}{d \lambda}(r)=-\left(r_{0}+\varepsilon-r\right)-2 \int_{r}^{r_{0}+\varepsilon} \\
& \frac{d \theta}{d \lambda}\left(\omega \frac{(J+1 / 2)}{r} \cos 2 \theta-m \sin 2 \theta\right) d r,
\end{aligned}
$$

from which by iteration we may deduce

$$
\frac{d \theta}{d \lambda}=0(\varepsilon) \quad\left(r_{0}-\varepsilon \leqq r<r_{0}+\varepsilon\right) .
$$
Using (10) we may make similar estimates of $R$ and $\frac{d R}{d \lambda}$. For the matrix $M_{\delta}\left(\varepsilon, r_{0}, g\right)$
defined respectively by

$$
\left(\begin{array}{l}
\phi\left(r_{0}-\varepsilon\right) \\
\psi\left(r_{0}-\varepsilon\right)
\end{array}\right)=M_{\delta}\left(\varepsilon, r_{0}, g\right)\left(\begin{array}{l}
\phi\left(r_{0}+\varepsilon\right) \\
\psi\left(r_{0}-\varepsilon\right)
\end{array}\right)
$$

we have

$$
M_{\delta}\left(\varepsilon, r_{0}, g\right)=M_{\delta}(g)+0\left(\varepsilon / r_{0}\right),
$$

where

$$
M_{\delta}(g)=\left(\begin{array}{rr}
\cos g & \sin g \\
-\sin g & \cos g
\end{array}\right) .
$$

We suppress the dependence on $\lambda$, but note that

$$
\frac{d}{d \lambda} M_{\delta}\left(\varepsilon, r_{0}, g\right)=0(\varepsilon) \text {. }
$$

We shall also require an estimate, in the case $V(r) \equiv 0$ and in the limit $a \rightarrow 0$, for the matrices $M_{1}(\lambda, a)$ and $M_{2}(\lambda, a)$, defined respectively by

$$
\begin{aligned}
& \left(\begin{array}{l}
\phi\left(c a^{2}\right) \\
\psi\left(c a^{2}\right)
\end{array}\right)=M_{1}(\lambda, a)\left(\begin{array}{l}
\phi(a) \\
\psi(a)
\end{array}\right), \\
& \left(\begin{array}{l}
\phi\left(c^{2} a^{3}\right) \\
\psi\left(c^{2} a^{3}\right.
\end{array}\right)=M_{2}(\lambda, a)\left(\begin{array}{l}
\phi\left(c a^{2}\right) \\
\psi\left(c a^{2}\right)
\end{array}\right),
\end{aligned}
$$

where $c$ is a constant. 
We take the simplest case $\omega=-1, J=1 / 2$. Solving Equation (4) and expanding in powers of $a$ we find

$$
M_{1}(\lambda, a)=\left(\begin{array}{cc}
c a+0\left(a^{2}\right) ; & -(\lambda+m) a+0\left(a^{2}\right) \\
\frac{(\lambda-m)}{3 c}+0\left(a^{2}\right) ; & \frac{1}{c a}+0(a)
\end{array}\right)
$$

and

$$
M_{2}(\lambda, a)=\left(\begin{array}{cc}
c a+0\left(a^{4}\right) ; & -c(\lambda+m) a^{2}+0\left(a^{3}\right) \\
\frac{(\lambda-m)}{3} a+0\left(a^{3}\right) ; & \frac{1}{c a}+0\left(a^{3}\right)
\end{array}\right) .
$$

We now have

$$
M(\lambda, a) \equiv M_{2}(\lambda, a) M_{\delta}(\pi / 2) M_{1}(\lambda, a)=M(\lambda)+0(a)
$$

and

$$
\frac{d M(\lambda, a)}{d \lambda}=\frac{d M(\lambda)}{d \lambda}+0(a)
$$

where

$$
M(\lambda)=\left(\begin{array}{cc}
0 & 1 \\
-1 & \frac{2(2 \lambda+m)}{3 c}
\end{array}\right)
$$

$M(\lambda, a)$ is the matrix relating $\left(\begin{array}{l}\phi \\ \psi\end{array}\right)$ at $r=c^{2} a^{3}$ and at $r=a$, for the potential $\frac{\pi}{2} \delta\left(r-c a^{2}\right)$.

Now let $w(r)$ satisfy

$$
\begin{aligned}
& w(r)=0 \text { for }|r|>1, \text { and } \\
& \int_{-1}^{1} w(r) d r=1 .
\end{aligned}
$$

Then the estimates we have made for $\delta$-approximating potentials show that (22) and (23) are also satisfied for the potential $\frac{\pi}{2} a^{-5} w\left(\frac{r-c a^{2}}{a^{5}}\right)$, where $M(\lambda, a)$ is defined by the condition

$$
\left(\begin{array}{l}
\phi\left(c^{2} a^{3}\right) \\
\psi\left(c^{2} a^{3}\right)
\end{array}\right)=M(\lambda, a)\left(\begin{array}{l}
\phi(a) \\
\psi(a)
\end{array}\right)
$$

For a potential $\frac{\pi}{2} a^{-(4+\varepsilon)} w\left(\frac{r-c a^{2}}{a^{4+\varepsilon}}\right)$, with $\varepsilon>0,(22)$ and (23) are satisfied to order $a^{\varepsilon}$.

Now define the potential $V$ by

$$
V(r)=\frac{\pi}{2} \sum_{k=1}^{\infty} a_{k}^{-5} w\left(\frac{r-c a_{k}^{2}}{a_{k}^{5}}\right),
$$

where

$$
c a_{k+1}=\left(c^{2} a\right)^{3^{k}}
$$


and

$$
c^{2} a<1 .
$$

With the potential defined by (25), we have

$$
\left(\begin{array}{l}
\phi\left(a_{k+1}\right) \\
\psi\left(a_{k+1}\right)
\end{array}\right)=M_{(k)}(\lambda)\left(\begin{array}{l}
\phi\left(a_{k}\right) \\
\psi\left(a_{k}\right)
\end{array}\right)
$$

where

$$
M_{(k)}(\lambda)=M(\lambda)+0\left(a_{k}\right)
$$

and

$$
\frac{d M_{(k)}(\lambda)}{d \lambda}=\frac{d M(\lambda)}{d \lambda}+O\left(a_{k}\right)
$$

(Note that $a_{k+1}=c^{2} a_{k}^{3}$.)

$V(r)$ is an "approximation" to the potential

$$
\frac{\pi}{2} \sum_{k=1}^{\infty} \delta\left(r-c a_{k}^{2}\right)
$$

For $-\frac{(m+3 c)}{2}<\lambda<\frac{(-m+3 c)}{2}$ the matrix $M(\lambda)$ defined by (24) has complex eigenvalues $e^{ \pm i \mu(\lambda)}$, with $\cos \mu(\lambda)=\frac{2 \lambda+m}{3 c}$. We may summarise as follows the conclusions which may be drawn from the estimates of $M_{(k)}(\lambda)$ (for the method of proof see [11]), where now $\psi(r)$ plays the role of $\phi^{\prime}(r)$ :

$$
\underline{I:\left|\lambda+\frac{m}{2}\right|<\frac{3 c}{2}}
$$

(i) $W(\lambda)=\lim _{n \rightarrow \infty}(M(\lambda))^{-n} M_{(n)}(\lambda) M_{(n-1)}(\lambda) \ldots M_{(1)}(\lambda)$

exists, is differentiable with respect to $\lambda$, and has uniformly bounded inverse in any closed subinterval.

(ii) Every solution $\left(\begin{array}{l}\phi \\ \psi\end{array}\right)$ of (4) satisfies

$$
\left(\begin{array}{l}
\phi\left(a_{n+1}\right) \\
\psi\left(a_{n+1}\right)
\end{array}\right) \sim\left(e^{i n \mu} E_{+}+e^{-i n \mu} E_{-}\right) W\left(\begin{array}{l}
\phi\left(a_{1}\right) \\
\psi\left(a_{1}\right)
\end{array}\right),
$$

where $E_{ \pm}$are the (two dimensional) projection matrices corresponding to the eigenvalues $e^{ \pm i \mu}$.

There is no (non-trivial) solution in $\left[L_{2}\left(0, a_{1}\right)\right]^{2}$.

$$
\text { (iii) } \begin{aligned}
& \lim _{n \rightarrow \infty} \frac{1}{n} \int_{a_{n+1}}^{a_{1}}\left(\phi^{2}+\psi^{2}\right) d r \\
= & \frac{2}{3 c \sin ^{2} \mu}\left[W\left(\begin{array}{l}
\phi\left(a_{1}\right) \\
\psi\left(a_{1}\right)
\end{array}\right)\right]^{T}\left(\begin{array}{cc}
1 & -\cos \mu \\
-\cos \mu & 1
\end{array}\right)\left[W\left(\begin{array}{l}
\phi\left(a_{1}\right) \\
\psi\left(a_{1}\right)
\end{array}\right)\right] .
\end{aligned}
$$


(iv) The spectrum of $H_{1}$ (within this interval) is absolutely continuous.

$$
I I:\left|\lambda+\frac{m}{2}\right|>\frac{3 c}{2} \text {. }
$$

In this case, $M(\lambda)$ has real eigenvalues. Again $W(\lambda)$ exists and satisfies (i) above. Moreover,

(ii) Every solution $\left(\begin{array}{l}\phi \\ \psi\end{array}\right)$ of (4) satisfies

$$
\left(\begin{array}{l}
\phi\left(a_{n+1}\right) \\
\psi\left(a_{n+1}\right)
\end{array}\right) \sim\left(\gamma^{n} E^{+}+\gamma^{-n} E^{-}\right) W\left(\begin{array}{l}
\phi\left(a_{1}\right) \\
\psi\left(a_{1}\right)
\end{array}\right),
$$

where $E^{ \pm}$are the projections corresponding to eigenvalues $\gamma$ and $\gamma^{-1}$, with $0<\gamma<1$.

If $H_{1}$ is defined by the boundary condition $\sin \beta \phi\left(a_{1}\right)-\cos \beta \psi\left(a_{1}\right)=0$, the eigenvalues of $H_{1}$ occur whenever $E^{-} W\left(\begin{array}{l}\cos \beta \\ \sin \beta\end{array}\right)=0$. For each value of $\lambda$ there is exactly one linearly independent solution of $(4)$ in $\left[L_{2}\left(0, a_{1}\right)\right]^{2}$.

(iii) For this range of values of $\lambda$, the spectrum of $H_{1}$ is purely discrete.

\section{Change of Coupling Constant}

We consider the effect on the spectral properties of $H_{1}$ of a change in coupling constant, replacing $\pi / 2$ by $g$ in (25). In that case, instead of (28), the matrix $M_{(k)}(\lambda)$, defined by (27), now satisfies

$$
M_{(k)}(\lambda)=\left(\begin{array}{cc}
(1 / 3) a_{k}(\lambda-m) \sin g+0\left(a_{k}^{2}\right) ; & \sin g+0\left(a_{k}\right) \\
\frac{(\lambda-m) \cos g}{3 c^{2} a_{k}}+0(1) ; & \frac{\cos g}{c^{2} a_{k}^{2}}+0\left(\frac{1}{a_{k}}\right)
\end{array}\right) .
$$

For simplicity, we take $\cos g>0, \sin g>0$, and choose positive constants $d_{1}, d_{2}$, such that $d_{1}<\cos g<d_{2}$.

Using mathematical induction, we may show that a solution $\left(\begin{array}{l}\phi \\ \psi\end{array}\right)$ of (4) exists such that

$$
\left.\begin{array}{l}
\frac{d_{1}^{k-1} \sin g}{2\left(c^{2} a\right)^{\beta \cdot 3^{k-1}}}<\phi\left(a_{k+1}\right) \frac{2 d_{2}^{k-1} \sin g}{\left(c^{2} a\right)^{\alpha \cdot 3^{k-1}}} \\
\frac{d_{1}^{k}}{\left(c^{2} a\right)^{\beta^{\prime} \cdot 3^{k}}}<\psi\left(a_{k+1}\right)<\frac{d_{2}^{k}}{\left(c^{2} a\right)^{\alpha^{\prime} \cdot 3^{k}}}
\end{array}\right\}, \quad\left(k \geqq k_{0}\right)
$$

where $\alpha, \alpha^{\prime}>1$ and $\beta, \beta^{\prime}<1$.

$\alpha, \alpha^{\prime}, \beta, \beta^{\prime}$ depend on $k$; in fact

$$
\begin{array}{ll}
\alpha_{k+1}^{\prime}=(1 / 3)\left(\alpha_{k}^{\prime}+2\right), & \beta_{k+1}^{\prime}=(1 / 3)\left(\beta_{k}^{\prime}+2\right), \\
\alpha_{k+1}=\alpha_{k}^{\prime}, & \beta_{k+1}=\beta_{k}^{\prime} .
\end{array}
$$

Hence $\alpha, \alpha^{\prime}, \beta, \beta^{\prime}$ converge to 1 as $k \rightarrow \infty$. The induction begins with an initial (large) value of $k$, with values of $\alpha, \alpha^{\prime}, \beta, \beta^{\prime}$ close to 1 .

$$
\text { Note that } \frac{\phi\left(a_{k+1}\right)}{\psi\left(a_{k+1}\right)}<2 \frac{\sin g}{d_{2}}\left(\frac{d_{2}}{d_{1}}\right)^{k}\left(c^{2} a\right)^{\left(\beta^{\prime}-\alpha / 3\right) 3^{k}} \rightarrow 0 \text { as } k \rightarrow \infty \text {. }
$$


For this solution, we have, for any $\varepsilon>0$ and for $k$ sufficiently large,

$$
\left.\begin{array}{l}
\frac{1}{\left(c^{2} a\right)^{(1-\varepsilon) 3^{k-1}}}<\phi\left(a_{k+1}\right)<\frac{1}{\left(c^{2} a\right)^{(1+\varepsilon) 3^{k-1}}} \\
\frac{1}{\left(c^{2} a\right)^{(1-\varepsilon) 3^{k}}}<\psi\left(a_{k+1}\right)<\frac{1}{\left(c^{2} a\right)^{(1+\varepsilon) 3^{k}}}
\end{array}\right\} .
$$

Writing now $\left(\begin{array}{l}\phi\left(a_{k+1}\right) \\ \psi\left(a_{k+1}\right)\end{array}\right)=\left(\begin{array}{l}x_{k} \\ y_{k}\end{array}\right)$, so that $\left(\begin{array}{l}x_{k+1} \\ y_{k+1}\end{array}\right)=M_{(k+1)}\left(\begin{array}{l}x_{k} \\ y_{k}\end{array}\right)$, a second solution of this recurrence relation is given by the following

Lemma. Let $X_{k}=-1 / y_{k}+x_{k} \sum_{n=k}^{\infty} \frac{M_{(n+1)}^{21}}{y_{n} y_{n+1}}$, and

$$
Y_{k}=y_{k} \sum_{n=k}^{\infty} \frac{M_{(n+1)}^{21}}{y_{n} y_{n+1}}
$$

where $M_{(n+1)}^{i j}$ stands for the $i, j$ element of $M_{(n+1)}(\lambda)$. Then

$$
\left(\begin{array}{c}
X_{k+1} \\
Y_{k+1}
\end{array}\right)=M_{(k+1)}\left(\begin{array}{c}
X_{k} \\
Y_{k}
\end{array}\right) \text {. }
$$

Proof.

$$
\begin{aligned}
M_{(k+1)}^{11} X_{k}+M_{(k+1)}^{12} Y_{k}= & -\frac{M_{(k+1)}^{11}}{y_{k}}+x_{k+1} \sum_{n=k}^{\infty} \frac{M_{(n+1)}^{21}}{y_{n} y_{n+1}} \\
= & -\frac{1}{y_{k+1}}+x_{k+1} \sum_{n=k+1}^{\infty} \frac{M_{(n+1)}^{21}}{y_{n} y_{n+1}} \\
& -\frac{M_{(k+1)}^{11}}{y_{k}}+\frac{x_{k+1} M_{(n+1)}^{21}}{y_{k} y_{k+1}}+\frac{1}{y_{k+1}} .
\end{aligned}
$$

Now $\operatorname{det} M_{(k+1)}^{i j}=1$, so that

$$
\begin{aligned}
& -M_{(k+1)}^{11}\left(M_{(k+1)}^{21} x_{k}+M_{(k+1)}^{22} y_{k}\right) \\
& +M_{(k+1)}^{21}\left(M_{(k+1)}^{11} x_{k}+M_{(k+1)}^{12} y_{k}\right)+y_{k}=0 .
\end{aligned}
$$

I.e. $-M_{(k+1)}^{11} y_{k+1}+M_{(k+1)}^{21} x_{k+1}+y_{k}=0$, so that on dividing throughout by $y_{k} y_{k+1}$ we find

$$
M_{(k+1)}^{11} X_{k}+M_{(k+1)}^{12} Y_{k}=X_{k+1} .
$$

The second component of (32) follows similarly.

We can define a solution of (4) by $\Phi\left(a_{k+1}\right)=X_{k}, \Psi\left(a_{k+1}\right)=Y_{k}$, and using the Lemma together with the estimates (31) of $x_{k}, y_{k}$, and the estimate (30) for $M_{(k+1)}^{21}$, we have

$$
\begin{aligned}
& \Phi\left(a_{k+1}\right)=0\left(\left(c^{2} a\right)^{(1-\varepsilon) 3^{k}}\right) \\
& \Psi\left(a_{k+1}\right)=0\left(\left(c^{2} a\right)^{(2-\varepsilon) 3^{k}}\right) \quad \text { as } k \rightarrow \infty .
\end{aligned}
$$


Integrating (10) between $a_{k+1}$ and $q$, where $a_{k+1} \leqq q \leqq a_{k}$ we find that the elements of the matrix sending $\left(\begin{array}{l}\Phi\left(a_{k+1}\right) \\ \Psi\left(a_{k+1}\right)\end{array}\right)$ to $\left(\begin{array}{l}\Phi(q) \\ \Psi(q)\end{array}\right)$ are of order $\frac{a_{k}}{a_{k+1}}$ at most. Since $\frac{a_{k}}{a_{k+1}}$ $=\frac{\left(c^{2} a\right)^{3^{k-1}}}{\left(c^{2} a\right)^{3^{k}}}$, this gives

$$
\Phi(q)=0\left(\left(c^{2} a\right)^{(1-\varepsilon) 3^{k-1}}\right)=0\left(a_{k+1}^{(1-\varepsilon) / 3}\right),
$$

with a similar estimate for $\Psi(q)$.

But always $q \geqq a_{k+1}$, so that we now have

$$
\Phi(r)=0\left(r^{(1-\varepsilon) / 3}\right), \quad \Psi(r)=0\left(r^{4(1-\varepsilon) / 3}\right) .
$$

(These estimates are not the best possible.)

The results of Section (3) may now be used to define a resolvent for $H_{a}$, and noting that for every solution $\left(\begin{array}{l}\phi \\ \psi\end{array}\right)$ we have $\phi=0\left(\frac{1}{r}\right), \psi=0\left(\frac{1}{r}\right)$, we deduce that the resolvent is Hilbert Schmidt.

Hence with a general value $g$ of the coupling constant the spectrum of $H_{a}$ is purely discrete; the only exceptional cases are $g=\frac{\pi}{2}+n \pi(n=0, \pm 1, \pm 2, \ldots)$. Our conclusions concerning asymptotic completeness may be summarised as follows:

Theorem 4. Let

$$
\begin{aligned}
V(r) & =g \sum_{k=1}^{\infty} a_{k}^{-5} w\left(\frac{r-c a_{k}^{2}}{a_{k}^{5}}\right), & & r<a_{1}, \\
& =0, & & r>a_{1},
\end{aligned}
$$

where $c a_{k+1}=\left(c^{2} a\right)^{3^{k}}, c^{2} a<1$, and $w(r)$ satisfies

$$
\begin{aligned}
& w(r)=0, \quad|r|>1, \\
& \int_{-1}^{1} w(r) d r=1 .
\end{aligned}
$$

Let $H$ be the (unique) self-adjoint extension, in $\left[L_{2}(0, \infty)\right]^{2}$ of $\tau$ [given by Equation (3)] and let $H_{0}$ be the free Dirac Hamiltonian.

Then the wave operators $\Omega_{ \pm}\left(H, H_{0}\right)$ exist and

(i) $g=\frac{\pi}{2}+n \pi, \quad m<3 c \Rightarrow$ range $\left(\Omega_{ \pm}\right) \neq$range $\left(\Omega_{-}\right), S=\Omega_{-}^{*} \Omega_{+}$is non-unitary, $M_{\Sigma}^{ \pm}(H)$ are non-trivial,

$M_{\Sigma}^{+}(H) \neq M_{\Sigma}^{-}(H), \sigma_{\text {a.c. }}(H)=\left(-\infty,\left(\frac{-m+3 c}{2}\right)\right) \cup(m, \infty)$.

(ii) $g=\frac{\pi}{2}+n \pi, m>3 c \Rightarrow \operatorname{range}\left(\Omega_{+}\right)=\operatorname{range}\left(\Omega_{-}\right) \neq M_{\text {a.c. }}(H), S$ is unitary, $M_{\bar{\Sigma}}^{ \pm}(H)$ are non-trivial, $M_{\Sigma}^{+}(H)=M_{\Sigma}^{-}(H)$,

$\sigma_{\text {a.c. }}(H)=(-\infty,-m) \cup\left(\frac{(-m+3 c)}{2}, \frac{(-m+3 c)}{2}\right) \cup(m, \infty)$.

(iii) $g \neq \frac{\pi}{2}+n \pi \Rightarrow \operatorname{range}\left(\Omega_{+}\right)=\operatorname{range}\left(\Omega_{-}\right)=M_{\text {a.c. }}(H), S$ is unitary, $M_{\Sigma}^{ \pm}(H)=\{0\}$, $\sigma_{\text {a.c. }}(H)=(-\infty,-m) \cup(m, \infty)$.

In all cases there is an absence of singular continuous spectrum. 
Remarks. (i) Taking $w(r) \geqq 0$, we have

$$
V(r) \geqq 0 \quad \text { and } \quad \int_{0}^{1} r^{\varepsilon} V(r) d r<\infty \quad(\varepsilon>0) .
$$

(ii) Replacing, in $V(r), a_{k}^{ \pm 5}$ by $a_{k}^{ \pm(4+\varepsilon)}$, the conclusions of the theorem hold with $V(r)=0\left(1 / r^{2+\varepsilon}\right)$.

\section{References}

1. Eckardt,K.-J.: Manuscripta math. 11, 359-371 (1974)

2. Eckardt,K.-J.: Math. Z. 139, 105-131 (1974)

3. Jorgens, K.: Perturbations of the Dirac operator. Lecture notes in mathematics, Vol. 280. BerlinHeidelberg-New York: Springer 1973

4. Kalf, H., Schmincke,U.-W., Walter,J., Wüst, R.: On the spectral theory of Schrödinger and Dirac operators with strongly singular potentials. Lecture notes in mathematics, Vol. 448. BerlinHeidelberg-New York: Springer 1975

5. Kupsch,J., Sandhas, W.: Commun. math. Phys. 2, 147-154 (1966)

6. Messiah, A. : Quantum mechanics, Vol. II. Amsterdam: North Holland 1966

7. Nenciu, G.: Commun. math. Phys. 48, 235-247 (1976)

8. Nenciu, G.: Helv. Phys. Acta 50,1-3 (1977)

9. Pearson,D.B. : Helv. Phys. Acta 47, 249-264 (1974)

10. Pearson, D. B.: Helv. Phys. Acta 48, 639-653 (1975)

11. Pearson,D.B.: Commun. math. Phys. 40, 125-146 (1975)

12. Pearson,D.B.: A generalisation of the Birman trace theorem. Funct. Anal. (to be published)

Communicated by J. Ginibre

Received June 13, 1977 
